\title{
Radiofrequency ablation therapy for primary breast cancer: expectations and problems as a novel breast conservation therapy
}

\author{
Hitoshi Tsuda
}

Published online: 21 October 2010

(C) The Japanese Breast Cancer Society 2010

Minimally invasive surgery is a current trend in the field of surgical oncology of the breast, and breast conservation therapy comprising lumpectomy/quadrantectomy with radiation and sentinel lymph node navigation surgery are now generally accepted as standard therapies. In addition, novel forms of minimally invasive surgery, such as radiofrequency ablation (RFA), accelerated partial breast irradiation (APBI), and cryosurgery, are now being considered as potential approaches for non-surgical treatment of breast cancer. The four papers in this issue focus on RFA as a treatment for patients with early breast cancers.

First, a pilot study of RFA therapy without surgical excision for $\mathrm{T} 1$ breast cancer was presented by Dr. Naohito Yamamoto and his colleagues at Chiba Cancer Center. For 29 patients RFA was performed with a Cool-tip RF system, with sentinel lymph node biopsy. They did not resect the ablated tumors, and reported in detail the postoperative follow-up, histopathological examination of tumor viability based on core needle biopsy specimens, and the adverse effects of RFA during a relatively long period of follow-up. Their findings indicated that RFA therapy appeared relevant and applicable for patients with small breast cancers.

Second, another phase I/II study to evaluate the safety and efficacy of RFA as a local therapy for 50 patients with early breast cancer was described by Dr. Takayuki Kinoshita and his colleagues at the National Cancer Center Hospital. They performed RFA under ultrasound guidance initially, and subsequently surgical resection of the tumor. In their series, $24(83 \%)$ of 29 patients with breast carcinomas

\section{H. Tsuda ( $\square)$}

Pathology and Clinical Laboratory Division,

National Cancer Center Hospital, 5-1-1 Tsukiji,

Chuo-ku, Tokyo 104-0045, Japan

e-mail: hstsuda@ncc.go.jp
$2.0 \mathrm{~cm}$ in size or smaller showed complete ablation of the tumor on the basis of histopathology and enzyme histochemistry. Five patients experienced adverse events including skin or muscle burns. On the basis of their study results, Kinoshita et al. concluded that RFA was a safe and promising minimally invasive treatment for small breast cancers with a tumor size of $2.0 \mathrm{~cm}$ or less, and without an extensive intraductal component (EIC).

Dr. Kunihiko Seki and colleagues at the JR Tokyo General Hospital reported on the histopathological effect of RFA with special reference to comparison between hematoxylin-eosin-stained sections and enzyme histochemistry using nicotinamide adenine dinucleotide (NADH) diaphorase staining. They described in detail the macroscopic and microscopic changes in the tumors and surrounding breast tissues. They provided a definition of a complete histopathological effect and evaluated the validity of this definition by a comparison with the results of the NADH diaphorase assay. However, they concluded that a larger number of cases should be examined in order to establish criteria for the histopathological effect of RFA on breast cancer.

The fourth presentation described a histopathological study to evaluate the therapeutic effect of RFA in patients with breast cancer. On the basis of histopathological examination of specimens resected from 28 patients who underwent RFA and subsequent surgical therapies for early breast cancer, Tsuda et al. reported the factors that influenced the histopathological completeness of the RFA effect. They concluded that a tumor size of $1.5 \mathrm{~cm}$ or smaller, strictly $1.0 \mathrm{~cm}$ or smaller, could be an indication for RFA if complete histological eradication is the desired outcome.

These four presentations will be informative for considering further large-scale prospective studies to validate 
the safety and efficacy of RFA therapy without surgical tumor resection for patients with early breast cancer. Until such clinical studies have been performed, it will not be possible to establish standard criteria for histopathological evaluation of the therapeutic effect or for clarifying the timing of NADH diaphorase staining in a clinical setting. 